\title{
Implementation of Predictive Control in a Three-Phase Neutral Point Clamped Inverter
}

\author{
Ricardo Enrique Pérez-Guzmán, Felipe Herrera, Marco Rivera, José Riveros and Patrick W. Wheeler
}

\begin{abstract}
In recent years, multilevel converters have established themselves as an efficient technology for the conversion of medium and high power. This research presents a NPC multilevel inverter topology, using two new control strategies: classical predictive control and fixed frequency predictive control. The results allow to verify the performance of each of the control strategies, which can be selected according to the application needs.
\end{abstract}

Keywords-Neutral point clamped inverter (NPC), model predictive control, fixed frequency, variable frequency.

\section{INTRODUCCIÓN}

Los convertidores multinivel son en la actualidad una clara opción para la integración de fuentes renovables, en aplicaciones de alta, media y baja potencia [1]. Al aumentar el número de conmutadores dentro de su estructura, estos proporcionan grados de libertad suplementarios en la modulación con respecto a los convertidores clásicos de dos niveles. Esta ventaja puede ser aprovechada para el control del voltaje en los capacitores; la reducción de las pérdidas, o la distorsión armónica de las tensiones de salida [2].

Existen tres topologías fundamentales de convertidores multinivel: NPC (condensadores conectados al punto neutro), las topologías en puente $\mathrm{H}$ y de condensadores flotantes. De ellas, sin dudas la topología más utilizada es NPC [3], debido a la reducción de la frecuencia de conmutación y el nivel de potencia de los semiconductores. Sin embargo, a pesar de su popularidad, todavía es necesario profundizar en la reducción del número de conmutaciones y con ello las pérdidas asociadas a este fenómeno [4].

La posibilidad de manejar bajos y altos voltajes, hace del NPC un inversor adecuado para la transmisión de energía limpia a la red, como la requerida por las microredes eléctricas. Otra de sus aplicaciones lo ubican como elemento fundamental en aplicaciones V2G (Vehicle-to-Grid), o V2H (Vehicleto-Home) [5]. Además, estos convertidores pueden generar corrientes, o incluso voltajes sinusoidales con mucho menor contenido armónico como se afirma en [6].

En el inversor NPC, la tensión del enlace de DC es dividida por dos condensadores, donde el punto medio $O$, se define

Ricardo Enrique Pérez-Guzmán, (Doctorado en Sistemas de Ingeniería), Departamento de Ciencias de la Computación, Facultad de Ingeniería, Universidad de Talca, Curicó, Chile (e-mail: riperez@utalca.cl).

Marco Rivera y José Riveros, Centro Tecnológico de Conversión de Energías, Facultad de Ingeniería, Universidad de Talca, Curicó, Chile (e-mail: marcoriv@utalca.cl, joservs@gmail.com).

Patrick W. Wheeler, Faculty of Science and Engineering, Department of Electrical and Electronic Engineering, University of Nottingham, Nottingham NG7 2RD U.K. (e-mail: pat.wheeler@ nottingham.ac.uk).

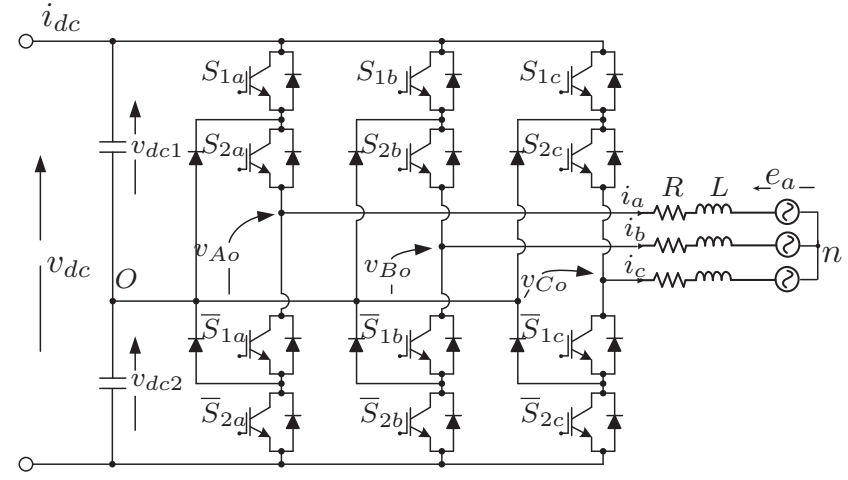

Fig. 1. Circuito de un inversor NPC trifásico conectado a una carga resistivainductiva y a la red eléctrica.

como el punto neutro. La tensión de salida en cada una de las fases, puede tomar tres niveles, $V_{d c} / 2,0 \mathrm{y}-V_{d c} / 2$ como se muestra en el circuito de la Fig. 1. Para las tres fases del inversor, se generan 27 estados de conmutación, que producen 19 vectores de voltaje diferentes. Para evitar el corto circuito, se definen los siguientes estados válidos: Positivo $(P)$, donde sólo conducen los switches $S_{1 x}$ y $S_{2 x}$, Cero $(O)$, estado en el cual conducen los switches intermedios $S_{2 x}$ y $\bar{S}_{1 x}$; Negativo $(N)$, en el cual conducen los switches $\bar{S}_{1 x}$ y $\bar{S}_{2 x}$ mientras $S_{1 x}$ y $S_{2 x}$ permanecen abiertos. En la Fig. 2 se muestran los vectores de voltaje generados, de acuerdo a la posible combinación de los switches.

La necesidad de interconectar inversores multinivel a la red, con estrategias de control eficientes y la menor cantidad de pérdidas, ha sido la principal motivación de esta investigación. En ella, se proponen dos nuevas estrategias de control predictivo basado en modelos (control predictivo a frecuencia variable y control predictivo a frecuencia fija), en un convertidor NPC. Además, de acuerdo a la literatura [7], ambas estrategias son similares, en cambio, la estrategia a frecuencia fija permite una disminución del rizado de la corriente de salida. Por este motivo, se comparan de acuerdo al error absoluto respecto a la corriente de salida y la distorsión armónica total y se destacan las ventajas de cada una de ellas.

\section{Modelo Del SISTEMA}

De acuerdo al diagrama del convertidor mostrado en la Fig. 1 y luego de aplicar las leyes de Kirchhoff y la Transformada de Clark, se obtiene el voltaje de salida en coordenadas $\alpha \beta$. 


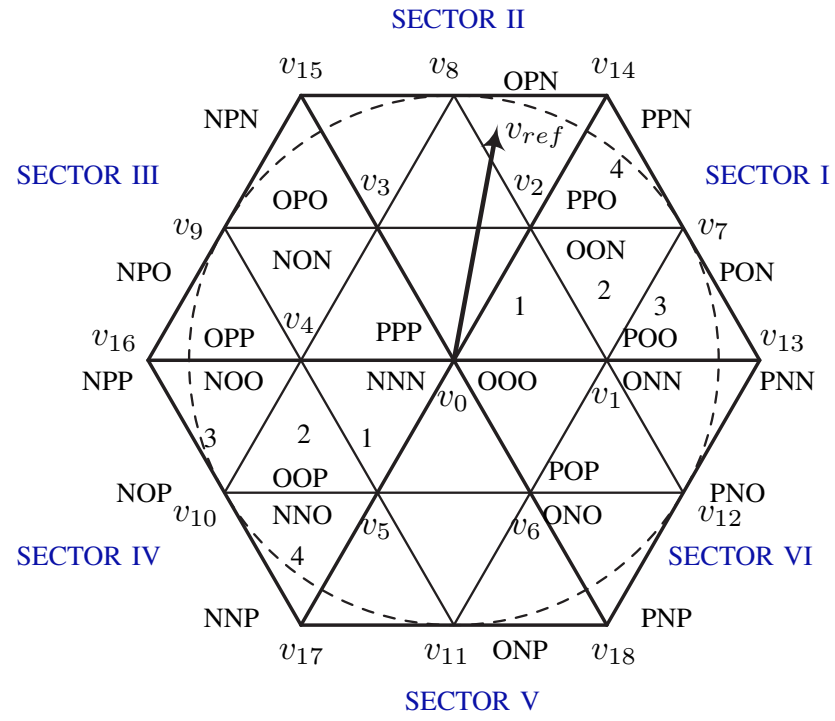

Fig. 2. Posibles vectores de voltaje y estados de conmutación generados por el inversor de tres niveles NPC (tomado de [4])

$$
v_{\alpha \beta}=R i_{\alpha \beta}+L \frac{d i_{\alpha \beta}}{d t}+e_{\alpha \beta}
$$

donde $R$ es la resistencia, $L$ es la inductancia, $e_{\alpha \beta}$ es el voltaje de la red, $i_{\alpha \beta}$, es la corriente y $v_{\alpha \beta}$ es el voltaje generado por el inversor respecto al punto medio del enlace DC.

Asumiendo que los voltajes a través de los dos capacitores del bus de DC son iguales, se puede afirmar que el voltaje en el punto neutro $O$, es igual a cero. En consecuencia, los voltajes de salida del convertidor se pueden expresar como:

$$
v_{x o}=S_{x} \frac{V_{d c}}{2}
$$

donde la función de conmutación $S_{x}(x=a, b, c)$ puede tomar los valores de 1, 0 ó -1 de acuerdo a los estados de conmutación de los switches, en cada una de las piernas.

Definiendo $T_{s}$ como el período de muestreo del convertidor y utilizando la aproximación de Euler hacia adelante, el análisis dinámico de las corrientes de carga del sistema se obtiene a partir de:

$$
\begin{gathered}
\frac{d i_{\alpha \beta}}{d t} \approx \frac{i_{\alpha \beta}(k+1)-i_{\alpha \beta}(k)}{T_{s}} \\
i_{\alpha \beta}(k+1)=\left(1-\frac{R T_{s}}{L}\right) i_{\alpha \beta}(k)+\frac{T_{s}}{L}\left[v_{\alpha \beta}(k)-e_{\alpha \beta}(k)\right]
\end{gathered}
$$

Para estimar la fuerza electro-motriz (FEM) se utilizan las mismas suposiciones que para el caso anterior, quedando:

$$
e_{\alpha \beta}(k-1)=v_{\alpha \beta}(k-1)-\frac{L}{T_{s}} i_{\alpha \beta}(k)-\left(R-\frac{L}{T_{s}}\right) i_{\alpha \beta}(k-1)
$$

La dinámica de los voltajes en los dos condensadores del enlace de DC se expresa en el dominio del tiempo como se muestra en las ecuaciones (6) y (7):

$$
v_{p}(t)=v_{p}(0)+\frac{1}{C} \int_{0}^{t} i_{p}(\tau) d \tau
$$

$$
v_{n}(t)=v_{n}(0)+\frac{1}{C} \int_{0}^{t} i_{n}(\tau) d \tau
$$

Utilizando la misma aproximación del método de Euler, los valores de $v_{p}(t), v_{n}(t)$ pueden obtenerse a partir de las siguientes ecuaciones:

$$
\begin{aligned}
& v_{p}(k+1)=v_{p}(k)+\frac{T_{s}}{C} i_{p}(k) \\
& v_{n}(k+1)=v_{n}(k)+\frac{T_{s}}{C} i_{n}(k)
\end{aligned}
$$

La función $S_{x 0}$, puede afectar a la corriente en el punto medio del bus de DC, de manera que:

$$
S_{x 0}\left\{\begin{array}{cc}
1 & \text { si } S_{x}=0 \\
0 & \text { resto }
\end{array} ; x=\{a, b, c\}\right.
$$

Por lo tanto, la corriente $i_{0}$ puede ser determinada en función de $S_{x 0}$ y las corrientes $i_{a}, i_{b}, i_{c}$ :

$$
i_{0}(k)=S_{a 0}(k) i_{a}(k)+S_{b 0}(k) i_{b}(k)+S_{c 0}(k) i_{c}(k)
$$

Finalmente, las ecuaciones (8) y (9), pueden ser reescritas en función de $i_{0}$ como:

$$
\begin{aligned}
& v_{p}(k+1)=v_{p}(k)+\frac{T_{s}}{2 C} i_{0}(k) \\
& v_{n}(k+1)=v_{n}(k)-\frac{T_{s}}{2 C} i_{0}(k)
\end{aligned}
$$

La predicción de $v_{p}$ y $v_{n}$ en el tiempo de muestreo $T_{s}(k+1)$, se puede lograr con las ecuaciones (12) - (13). Esta predicción se logra sin utilizar las corrientes que intervienen en cada uno de los condensadores. Sin embargo, el modelo predictivo presentado en [4], considera estas corrientes como:

$$
\begin{aligned}
& i_{c 1}(k)=i_{0}(k)-H_{1 a} i_{a}(k)-H_{1 b} i_{b}(k)-H_{1 c} i_{c}(k) \\
& i_{c 2}(k)=i_{0}(k)+H_{2 a} i_{a}(k)+H_{2 b} i_{b}(k)+H_{2 c} i_{c}(k)
\end{aligned}
$$

donde las variables $H_{1 x}$ y $H_{2 x}$ dependen del estado de conmutación definido como:

$$
\begin{aligned}
& H_{1 x}\left\{\begin{array}{lll}
1 & \text { si } S_{x}=+ \\
0 & \text { resto }
\end{array} \quad ; x=\{a, b, c\}\right. \\
& H_{2 x}\left\{\begin{array}{ccc}
1 & \text { si } S_{x}=- \\
0 & \text { resto }
\end{array} \quad ; x=\{a, b, c\}\right.
\end{aligned}
$$

A partir de las ecuaciones anteriores se proponen dos estrategias de control: el control predictivo basado en modelos con conjunto de control finito (FCS-MPC) y el control predictivo basado en modelos modulado $\left(\mathrm{M}^{2} \mathrm{PC}\right)$. Cada una de ellas es implementada de manera teórica y experimental y se comparan los resultados obtenidos.

\section{A. Control de corriente FCS-MPC con carga $R L$}

En esta estrategia los objetivos de control son: i) el seguimiento de la corriente de referencia, ii) el balance del voltaje en los capacitores del enlace de DC. Con esto, se determina una función de costo que incluye ambos objetivos 
de control (ecuación 18). De ella se desprenden las ecuaciones (19) referida a la corriente de seguimiento y la ecuación (20), referida al voltaje en los capacitores del enlace de DC.

$$
\begin{gathered}
g=\left|i_{\alpha}^{*}-i_{\alpha}^{p}\right|+\left|i_{\beta}^{*}-i_{\beta}^{p}\right|+\lambda_{d c}\left|v_{c 1}^{p}-v_{c 2}^{p}\right|+\lambda n_{c} \\
g_{s}(k+1)=\left(i_{\alpha}^{*}(k)-i_{\alpha}^{p}(k+1)\right)^{2}+\left(i_{\beta}^{*}(k+1)-i_{\beta}^{p}(k+1)\right)^{2} \\
g_{b}(k+1)=\left(v_{d c 1}(k+1)-v_{d c 2}(k+1)\right)^{2}
\end{gathered}
$$

La función de costos a evaluar para cada estado de conmutación es la suma de $g_{s}$ y $g_{b}$ multiplicadas por los factores de peso correspondientes.

$$
g(k+1)=g_{s}(k+1)+\lambda_{d c} g_{b}(k+1)
$$

En la Fig. 3 se presenta un diagrama de bloques de la estrategia FCS-MPC aplicada al convertidor NPC con carga $R L$ desarrollado en esta investigación. El diagrama muestra las variables que alimentan el modelo predictivo, los vectores de salida y la utilización de la función de costo.

\section{B. $M^{2} P C$ de corriente con carga $R L$}

El sistema, modelo matemático y objetivos de control de este caso son los mismos que los presentados en la sección anterior para el FCS-MPC de corriente. La función de costos tiene la misma estructura que para FCS-MPC, sin embargo, en este caso se evalúan los vectores $v_{0}, v_{1} \mathrm{y} v_{2}$, obteniendo las funciones $g_{0}, g_{1}$ y $g_{2}$, respectivamente. El conjunto de vectores que se aplica en el siguiente instante de muestreo es el que minimiza la función de costos (20). En la Fig. 4 se presenta un diagrama de bloques de la estrategia $\mathrm{M}^{2} \mathrm{PC}$ aplicada al convertidor NPC con carga $R L$. Las funciones de costos $g_{1} \mathrm{y}$ $g_{2}$ corresponden a la función de costo del vector activo $\mathbf{v}_{1} \mathrm{y}$ la función de costo del vector activo $\mathbf{v}_{2}$, respectivamente. El ciclo de trabajo de cada vector se calcula como $d_{j}=T_{j} / T_{s}$,

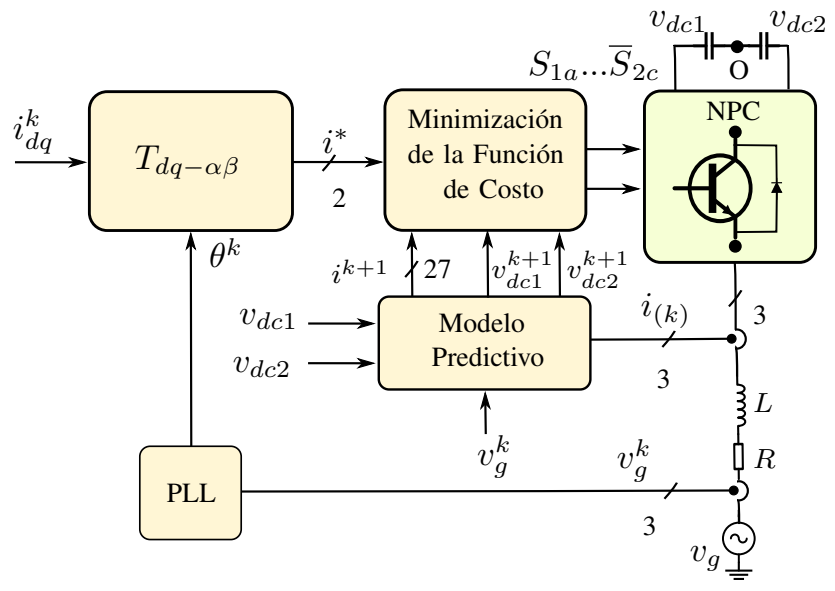

Fig. 3. Diagrama de bloques de la estrategia FCS-MPC para carga $R L$.

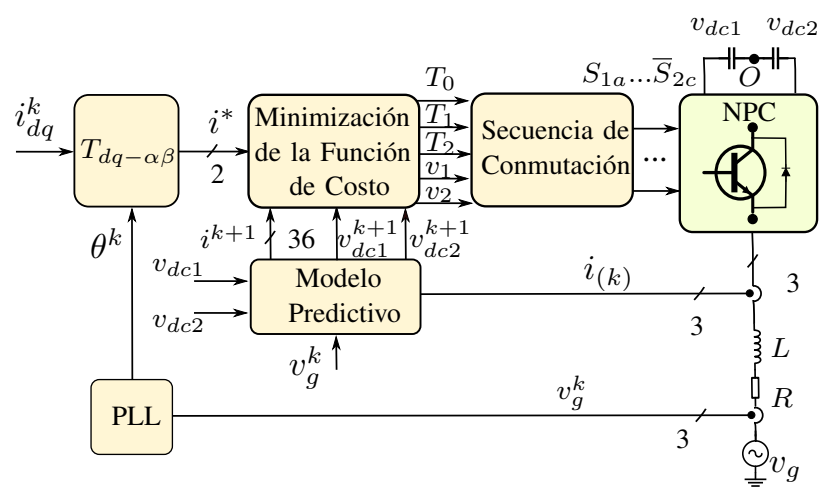

Fig. 4. Diagrama de bloques de la estrategia $\mathrm{M}^{2} \mathrm{PC}$ para carga $R L$

entonces los tiempos de aplicación de los vectores son:

$$
\begin{aligned}
& T_{0}=d_{0} T_{s}, \quad d_{0}=T_{s} g_{1} g_{2} /\left(g_{0} g_{1}+g_{1} g_{2}+g_{0} g_{2}\right) \\
& T_{1}=d_{1} T_{s}, \quad d_{1}=T_{s} g_{0} g_{2} /\left(g_{0} g_{1}+g_{1} g_{2}+g_{0} g_{2}\right) \\
& T_{2}=d_{2} T_{s}, \quad d_{2}=T_{s} g_{0} g_{1} /\left(g_{0} g_{1}+g_{1} g_{2}+g_{0} g_{2}\right)
\end{aligned}
$$

El ajuste del factor de peso en cada caso, se realiza forzando los factores de pesos a un valor cero $(\lambda=0)$. El segundo paso es establecer una medida que se utilizará para evaluar el rendimiento alcanzado por el factor de peso. En todos los casos, un valor debe ser relacionado con la variable primaria, y se denomina error actual, mientras por otro lado, es necesario un segundo valor, que será utilizado como referencia. Una vez que se definen las medidas se evalúa el comportamiento del sistema para $\lambda=0$ y se van aumentando gradualmente hasta que $\lambda=1$ [4]. De esta manera, se detendrá el incremento de $\lambda$ cuando el término secundario alcance el valor deseado para la aplicación específica, o hasta que la variable primaria exceda los valores permisibles.

\section{RESUltados}

El control predictivo de corriente aplicado al convertidor NPC incluyen los resultados de la simulación y resultados experimentales para la estrategia a frecuencia variable (FCSMPC) y a frecuencia fija $\left(\mathrm{M}^{2} \mathrm{PC}\right)$. Las frecuencias de muestreo consideradas son de $10[\mathrm{kHz}]$ y $20[\mathrm{kHz}]$ para el caso de la simulación y para el caso experimental $5 \mathrm{kHz}$. Además, se muestran resultados en estado estacionario y estado transitorio para 3 ciclos de la frecuencia fundamental $(50 \mathrm{~Hz})$, con un tiempo máximo de simulación de 0.1 [s]. Los criterios de rendimiento utilizados para la comparación entre controladores son la distorsión armónica (THD) y el error absoluto medio. La fórmula matemática del error absoluto medio es:

$$
e=\frac{\frac{\sum_{i=1}^{n}|| x_{0}|-| x_{r e f}||}{n}}{A_{\text {ref }}} \times 100
$$

donde $n$ es la cantidad de datos procesados, $x_{0}$ es el valor medido de la variable de interés, $x_{r e f}$ es el valor de referencia y $A_{r e f}$ es la amplitud de la referencia. En el caso de la corriente hacia la carga para el convertidor, el valor de la amplitud es 1 [A]. 


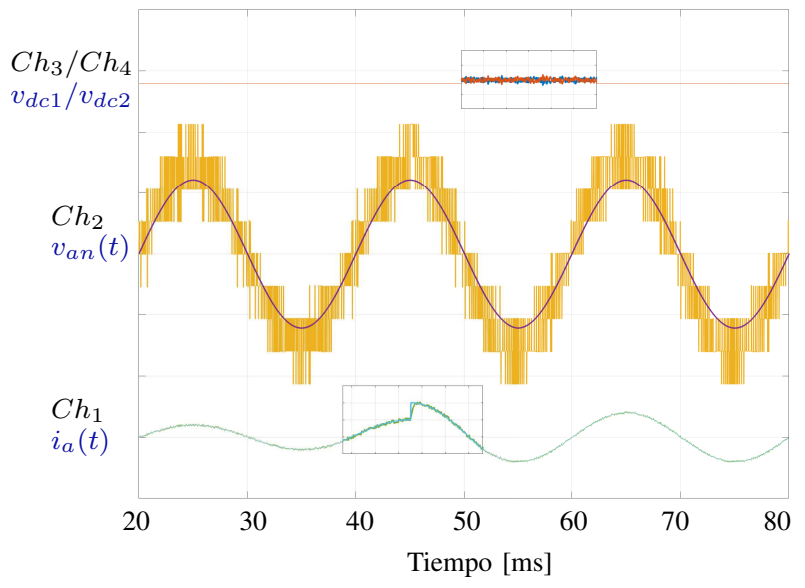

(a) Simulación del control predictivo a frecuencia variable (FCS-MPC), en estado transitorio.

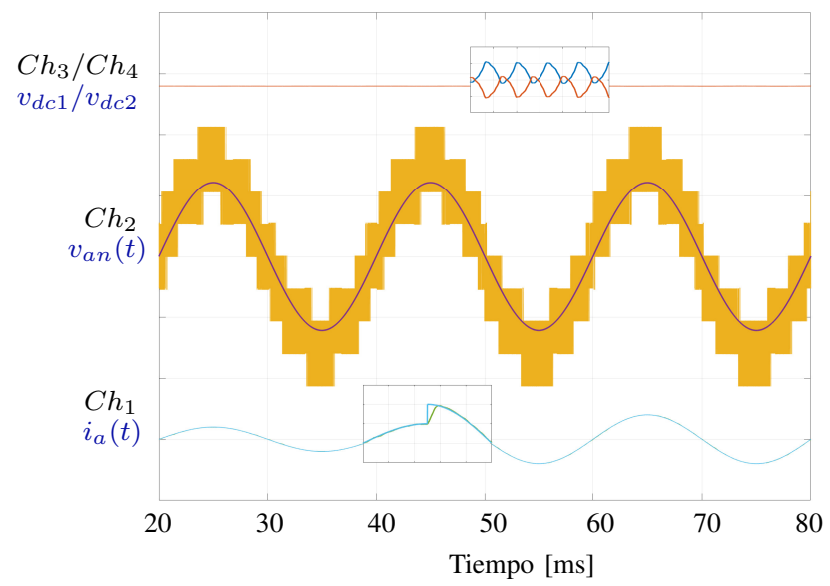

(b) Simulación del control predictivo a frecuencia fija $\left(\mathrm{M}^{2} \mathrm{PC}\right)$, en estado transitorio.

Fig. 5. Resultado de la simulación para el control predictivo a frecuencia variable (a) y a frecuencia fija (b) en estado transitorio.

\section{A. Resultados de la simulación}

Los valores fueron calculados para dos frecuencias de muestreo: $10 \mathrm{kHz}$ y $20 \mathrm{kHz}$, y se muestran con respecto a la corriente de salida de una de las fases del convertidor, de acuerdo a los parámetros mostrados en la Tabla I. En la Fig. $5(C h 3 / C h 4)$, se muestran los voltajes en los capacitores del enlace DC utilizando control FCS-MPC (a la izquierda) y el resultado del control con la estrategia $\mathrm{M}^{2} \mathrm{PC}$ (a la derecha). Se pudo comprobar que en ambos casos, se mantiene $v d c 1(t)$ y $v d c 2(t)$ en valores cercanos a los 80 [V], cumpliendo con el objetivo de control planteado de balancear el voltaje en los capacitores del enlace DC. En el caso del $\mathrm{M}^{2} \mathrm{PC}$, el desbalance de los capacitores es mayor con una diferencia de voltaje entre capacitores de 0.16 [V]. En la Fig. 5 se muestran el voltaje de fase $v_{a n}(t)[\mathrm{V}]$ para el convertidor conectado a la red y el voltaje de referencia $(C h 2)$. La corriente de referencia $i_{\text {ref }}(t)$ [A] y la corriente medida $i_{a}(t)$ [A], en la fase $a$ del convertidor con control FCS-MPC (izquierda) y control $\mathrm{M}^{2} \mathrm{PC}$ (derecha) se muestran en $C h 1$. En ambos casos se observa que el voltaje de la red y la corriente medida se mantienen en

TABLA I

PARÁMETROS DE LA SIMULACIÓN

\begin{tabular}{lll}
\hline Variables & Descripción & Valor \\
\hline$R$ & Resistencia & $10 \Omega$ \\
$L$ & Inductancia & $10^{-3} \mathrm{H}$ \\
$e$ & Amplitud pico de la FEM & $220 * \sqrt{2} \mathrm{~V}$ \\
$f_{r e d}$ & Frecuencia de referencia & $50 \mathrm{~Hz}$ \\
$V_{d c}$ & Voltaje del enlace de DC & $160 \mathrm{~V}$ \\
$e$ & Voltaje RMS de la FEM & $43 \mathrm{~V}$ \\
$T_{s}$ & Tiempo de muestreo & $50 \mu \mathrm{s} \mathrm{y} 100 \mu \mathrm{s}$ \\
$\lambda_{d c}$ & Factor de peso & 0.4 \\
\hline
\end{tabular}

fase y que siguen adecuadamente su referencia. Por otro lado, el voltaje de salida se mantiene en fase y se logran mejores resultados de predicción de corriente utilizando frecuencia fija de conmutación como se puede apreciar en la Fig. 5.

\section{B. Resultados experimentales}

La frecuencia de muestreo considerada es de $5[\mathrm{kHz}]$, debido a que el mínimo tiempo de cálculo conseguido para el algoritmo $\mathrm{M}^{2} \mathrm{PC}$ es de $130[\mu \mathrm{s}]$ en el procesador digital de señales (DSP). El valor de la amplitud de referencia para el cálculo del error absoluto medio es de 1 [A] y los parámetros experimentales para el control FCS-MPC y $\mathrm{M}^{2} \mathrm{PC}$ de corriente, con carga $R L$, se muestran en la Tabla II.

El procesador digital de señales (DSP) TMS320F28335 se encarga de ejecutar el algoritmo de control FCS-MPC, o $\mathrm{M}^{2} \mathrm{PC}$ y de enviar los estados de conmutación calculados a un arreglo de compuertas programables (FPGA), para su descomposición en las señales de disparo de los interruptores del convertidor. Además, se agrega un tiempo muerto entre interruptores complementarios, para evitar fallas del equipo por corto circuito. Los estados de conmutación se envían a través de los pines de entrada-salida digital (GPIO) de la DSP,

TABLA II

PARÁMETROS DE LA IMPLEMENTACIÓN EXPERIMENTAL DEL SISTEMA CON CARGA $R L$.

\begin{tabular}{lll}
\hline Variables & Descripción & Valor \\
\hline$V_{f}$ & Voltaje de la fuente $d c$ & $60[\mathrm{~V}]$ \\
$C_{d c}$ & Capacitor $d c$-link & $4700[\mu \mathrm{F}]$ \\
$R$ & Resistencia carga & $10[\Omega]$ \\
$L$ & Inductor carga & $0.01[\mathrm{H}]$ \\
$T_{s}$ & Tiempo de muestreo & $200[\mu \mathrm{s}]$ \\
$\lambda_{d c}$ & Factor de peso & 0.4 \\
\hline
\end{tabular}


hacia los conectores de entrada-salida digital de la FPGA, utilizando código binario. En la Fig. $6(C h 1)$, se muestran la corriente de referencia $i_{r e f}(t)$ y la corriente medida $i_{a}(t)$ en la fase $a$ del convertidor trabajando con control FCS-MPC (a la izquierda) y las mismas señales con el convertidor trabajando con control $\mathrm{M}^{2} \mathrm{PC}$ (a la derecha). Además, se muestra el voltaje de salida $(C h 2)$ y el voltaje de los capacitores del enlace DC (Ch3/Ch4).

\section{Comparación de resultados de control FCS-MPC y $M^{2} P C$}

Al comparar ambas estrategias es posible observar que se logra un buen seguimiento de la referencia, alcanzando los errores que se muestran en la Tabla III. La estrategia de control $\mathrm{M}^{2} \mathrm{PC}$ reduce el error aproximadamente a un tercio de lo obtenido por la estrategia FCS-MPC, cuando se trabaja a la misma frecuencia de muestreo.

TABLA III

ERROR ABSOLUTO MEDIO DE CORRIENTE PARA CONTROL FCS-MPC Y $\mathrm{M}^{2} \mathrm{PC}$ SIMULADO.

\begin{tabular}{ccc}
\hline Control & Frec. Muestreo & \% error $i_{a}$ \\
\hline FCS-MPC & $10[\mathrm{kHz}]$ & $8.73 \%$ \\
M $^{2}$ PC & $10[\mathrm{kHz}]$ & 2.58 \\
FCS-MPC & $20[\mathrm{kHz}]$ & $4.47 \%$ \\
M $^{2}$ PC & $20[\mathrm{kHz}]$ & $1.34 \%$ \\
\hline
\end{tabular}

La estrategia de control $\mathrm{M}^{2} \mathrm{PC}$ presenta una distorsión armónica aproximadamente 3.5 veces menor que la distorsión armónica de la corriente obtenida utilizando FCS-MPC, lo que permite obtener los mismos resultados utilizando frecuencias de muestreo menores. La Tabla IV muestra una comparación de los resultados experimentales del THD de corriente y voltaje, para el control el FCS-MPC y M $^{2}$ PC. Este desempeño relativamente pobre, con un seguimiento de referencias por encima del $10 \%$ se mejora ligeramente en otros puntos de operación. Además, es importante considerar que la frecuencia de muestreo es relativamente baja $(5[k H z])$, por lo que al aumentarla a valores por encima de $10[k H z]$, el error se reduce considerablemente.

$\mathrm{El}$ algoritmo de control $\mathrm{M}^{2} \mathrm{PC}$ presenta mejores resultados de THD de corriente frente al FCS-MPC cuando la amplitud es de 1 [A], pero sus resultados de THD de voltaje son mayores. Esto se debe a que la forma de onda del voltaje $v_{a n}$ con frecuencia fija es una forma de onda cuadrada debido al mayor número de conmutaciones forzadas de los interruptores y la

TABLA IV

DISTORSIÓN ARMÓNICA TOTAL DE $i_{a}$ Y van PARA EL CONTROL FCS-MPC Y M ${ }^{2}$ PC.

\begin{tabular}{cccc}
\hline Control & Amplitud $i_{a}$ & THD $i_{a}$ & THD $v_{a n}$ \\
\hline FCS-MPC & $1[\mathrm{~A}]$ & $16.12 \%$ & $101.34 \%$ \\
$\mathrm{M}^{2} \mathrm{PC}$ & $1[\mathrm{~A}]$ & $15.54 \%$ & $128.52 \%$ \\
FCS-MPC & $2[\mathrm{~A}]$ & $7.04 \%$ & $52.11 \%$ \\
$\mathrm{M}^{2} \mathrm{PC}$ & $2[\mathrm{~A}]$ & $9.70 \%$ & $68.07 \%$ \\
\hline
\end{tabular}

disminución de la frecuencia de muestreo. Adicionalmente, se puede decir que es necesaria una alta frecuencia de muestreo FCS-MPC para obtener un rendimiento similar a M2PC, en cuanto al THD se refiere. $\mathrm{M}^{2} \mathrm{PC}$ presenta también menor error absoluto medio de corriente, lo que demuestra su factibilidad. Cuando se evalúa el caso con una corriente de 2 [A], el controlador FCS-MPC obtiene $2 \%$ menos de distorsión de corriente y $10 \%$ menos de THD de voltaje con respecto al $\mathrm{M}^{2} \mathrm{PC}$, mostrando de manera experimental que el FCSMPC obtiene menor distorsión en algunos casos, como se muestra en la Fig 7 y la Fig. 8. El porcentaje de distorsión armónica total disminuye cuando se aumenta la referencia de corriente. El espectro armónico de voltaje del $\mathrm{M}^{2} \mathrm{PC}$, es la característica que posiciona a este controlador sobre el FCSMPC en aplicaciones donde se requieran de filtros de salida. Esto se debe a que los armónicos de voltaje se encuentran concentrados alrededor del armónico de orden 200 en el caso del $\mathrm{M}^{2} \mathrm{PC}$, lo que corresponde al doble de la frecuencia de muestreo. Por su parte, el espectro armónico de voltaje para el FCS-MPC muestra que los armónicos se encuentran distribuidos a lo largo del espectro, dificultando la tarea de diseño de filtros.

\section{CONCLUSIONES}

En esta investigación se presenta el diseño de un controlador predictivo de frecuencia de conmutación variable (FCS-MPC) $\mathrm{y}$ otro de frecuencia de conmutación fija $\left(\mathrm{M}^{2} \mathrm{PC}\right)$, para un convertidor NPC de tres niveles. Debido a las ventajas de este tipo de convertidor, su aplicación es muy importante para la integración de energías renovables. El método de control de corriente presentado no requiere ningún tipo de controlador lineal o técnica de modulación. El controlador $\mathrm{M}^{2} \mathrm{PC}$ muestra mejores resultados en cuanto al error absoluto y la distorsión armónica que el FCS-MPC, sin embargo, parece tener un menor rango de control en algunas aplicaciones, como lo demuestra el caso de control de voltaje. En el caso del control de voltaje, el controlador FCS-MPC es capaz de seguir exitosamente la referencia, para amplitudes de hasta 100 [V], mientras que el $\mathrm{M}^{2} \mathrm{PC}$ solo es capaz de seguir exitosamente la referencia hasta amplitudes de 85 [V]. Los resultados experimentales obtenidos para ambos controladores son acordes a los resultados teóricos obtenidos durante la simulación. Los resultados experimentales nos muestran que la diferencia entre $M^{2}$ PC y FCS-MPC no es extremadamente significativa como las simulaciones mostraban obteniendo valores de THD de corriente y de voltaje muy cercanos entre ellos. El $\mathrm{M}^{2} \mathrm{PC}$ presenta resultados superiores a los obtenidos por FCS-MPC. La gran ventaja del $\mathrm{M}^{2} \mathrm{PC}$ está en el espectro armónico del voltaje de salida, donde se observa que las componentes armónicas de mayor amplitud se concentran alrededor del doble de la frecuencia de conmutación, a diferencia del espectro armónico del FCS-MPC donde los armónicos se encuentra distribuidas a lo largo de todo el espectro. Esto última dificulta el diseño de los filtros de salida.

\section{Agradecimientos}

Los autores desean agradecer al Programa FONDECYT Regular a través del proyecto 1160690 y a la Beca de Doctorado 


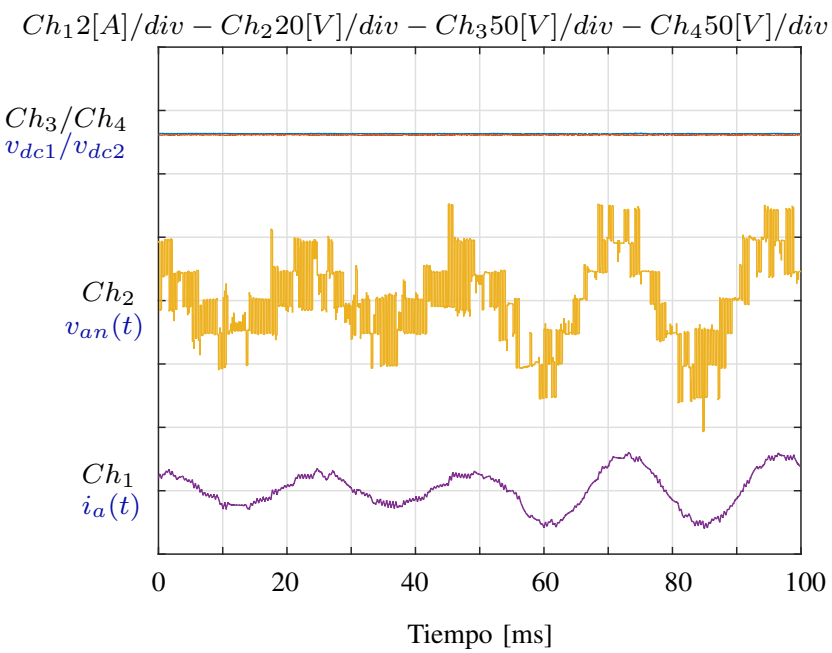

(a) Resultado experimental de control predictivo a frecuencia variable en estado transitorio. Corriente de referencia ante cambio en escalón de apmplitud desde $1[\mathrm{~A}]$ a $2[\mathrm{~A}]$ y frecuencia de $50[\mathrm{~Hz}]$.

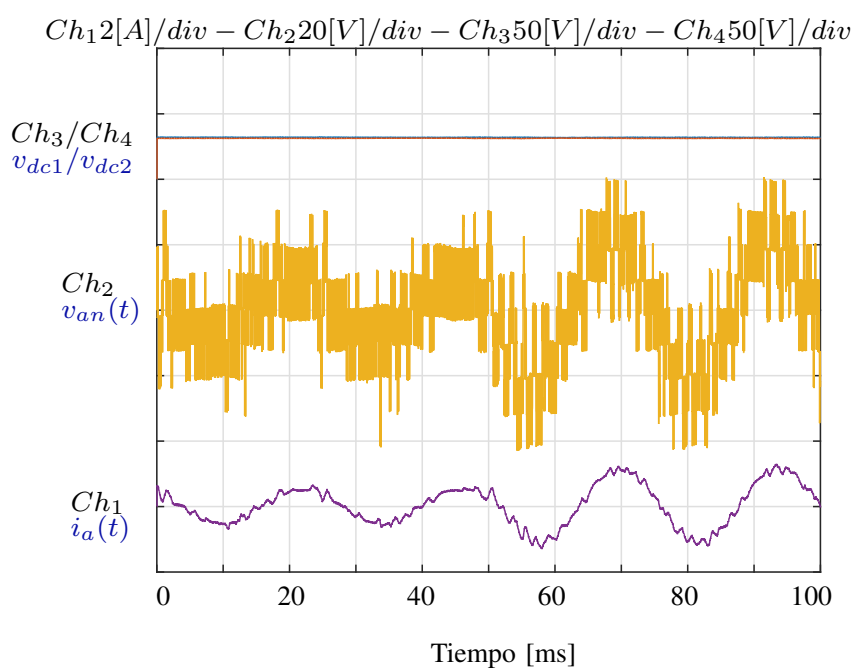

(b) Resultado experimental de control predictivo a frecuencia fija en estado transitorio. Corriente de referencia ante cambio en escalón de amplitud desde $1[\mathrm{~A}]$ a $2[\mathrm{~A}]$ y frecuencia de $50[\mathrm{~Hz}]$.

Fig. 6. Resultado experimental del control predictivo a frecuencia variable (a) y a frecuencia fija (b), en estado transitorio.
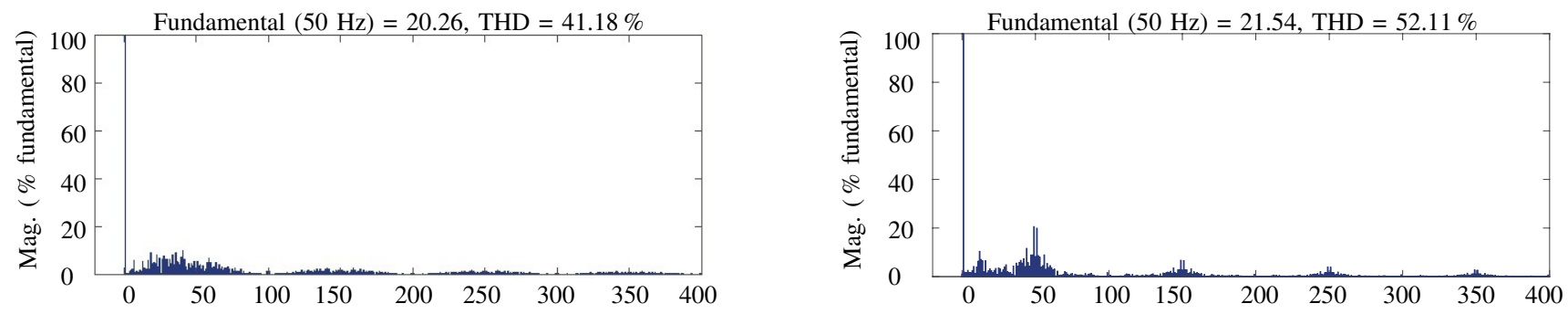

Fig. 7. Espectro de armónicos del voltaje de salida $v_{a n}(t)$ para los resultados en simulación (a la izquierda) y experimental (a la derecha), operando con una frecuencia de muestreo de $5[\mathrm{kHz}]$, control FCS-MPC y amplitud de $2[\mathrm{~A}]$.
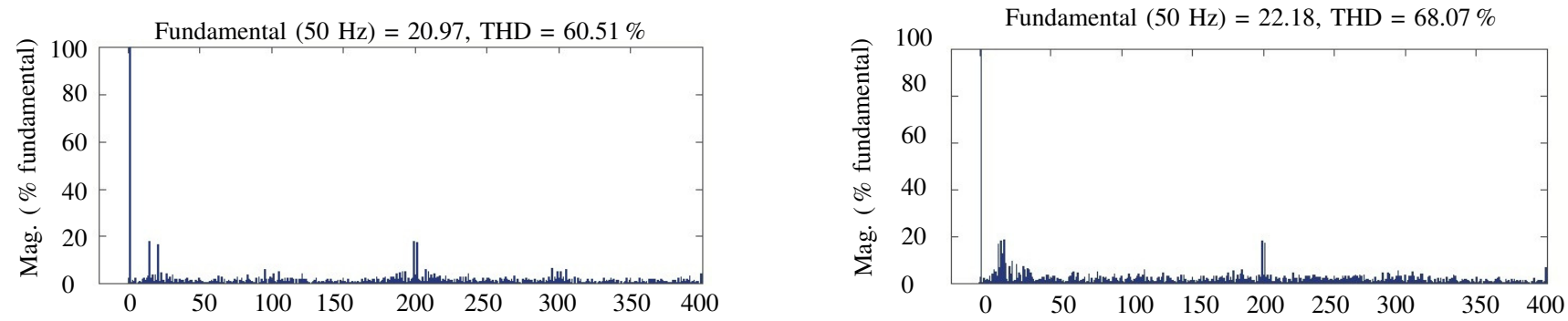

Fig. 8. Espectro de armónicos del voltaje de salida $v_{a n}(t)$ para los resultados en simulación (a la izquierda) y experimental (a la derecha), operando con una frecuencia de muestreo de $5[\mathrm{kHz}]$, control $\mathrm{M}^{2} \mathrm{PC}$ y amplitud de $2[\mathrm{~A}]$.

\section{Nacional CONICYT 2019, folio 21190709.}

\section{REFERENCIAS}

[1] J. S. M. Ali and V. Krishnaswamy, "An assessment of recent multilevel inverter topologies with reduced power electronics components for renewable applications," Renewable and Sustainable Energy Reviews, vol. 82, pp. 3379-3399, 2018.

[2] D. Mukherjee and D. Kastha, "A reduced switch hybrid multilevel unidirectional rectifier," IEEE Transactions on Power Electronics, vol. 34, no. 3, pp. 2070-2081, 2019.

[3] B. Malakondareddy, S. S. Kumar, N. A. Gounden, and I. Anand, "An adaptive pi control scheme to balance the neutral-point voltage in a solar pv fed grid connected neutral point clamped inverter," International Journal of Electrical Power \& Energy Systems, vol. 110, pp. 318-331, 2019.
[4] J. Rodriguez and P. Cortes, Predictive control of power converters and electrical drives. John Wiley Sons, 2012, vol. 40.

[5] A. Mortezaei, M. Abdul-Hak, and M. G. Simoes, "A bidirectional npcbased level 3 ev charging system with added active filter functionality in smart grid applications," in 2018 IEEE Transportation Electrification Conference and Expo (ITEC). IEEE, 2018, pp. 201-206.

[6] Y. Li, X. Yang, W. Chen, T. Liu, and F. Zhang, "Neutral-point voltage analysis and suppression for npc three-level photovoltaic converter in lvrt operation under imbalanced grid faults with selective hybrid svpwm strategy," IEEE Transactions on Power Electronics, vol. 34, no. 2, pp. 1334-1355, 2019.

[7] L. Tarisciotti, P. Zanchetta, A. Watson, S. Bifaretti, and J. C. Clare, "Modulated model predictive control for a seven-level cascaded h-bridge back-to-back converter," IEEE Transactions on industrial electronics, vol. 61, no. 10, pp. 5375-5383, 2014. 